\title{
Decay Versus Survival of a Localized State Subjected to Harmonic Forcing: Exact Results
}

\author{
A.Rokhlenko, O. Costin, J. L. Lebowitz* \\ Department of Mathematics, Rutgers University \\ Piscataway, NJ 08854-8019
}

\begin{abstract}
We investigate the survival probability of a localized 1-d quantum particle subjected to a time dependent potential of the form $r U(x) \sin \omega t$ with $U(x)=2 \delta(x-a)$ or $U(x)=2 \delta(x-a)-2 \delta(x+a)$. The particle is initially in a bound state produced by the binding potential $-2 \delta(x)$. We prove that this probability goes to zero as $t \rightarrow \infty$ for almost all values of $r, \omega$, and $a$. The decay is initially exponential followed by a $t^{-3}$ law if $\omega$ is not close to resonances and $r$ is small; otherwise the exponential disappears and Fermi's golden rule fails. For exceptional sets of parameters $r, \omega$ and $a$ the survival probability never decays to zero, corresponding to the Floquet operator having a bound state. We show similar behavior even in the absence of a binding potential: permitting a free particle to be trapped by harmonically oscillating delta function potential.
\end{abstract}

PACS: 03.65.Db; 03.65.Ge; 32.80.Fb

\section{Introduction}

Quantum systems subjected to strong external time dependent fields often show very complex behavior, e.g. the ionization probability of an atom may be a complicated function of the frequency, amplitude, pulse shape and other parameters of the field [1-4]. Such phenomena, which go beyond conventional perturbation theory in the field strength ( $r$ here), are readily seen in numerical solutions of the time dependent Schrödinger equation. There are also various approximate analytic methods which reproduce many experimental results $[5,6,7]$, but there is however no rigorous theory of such phenomena even for model systems. In this paper we describe new exact results for a toy model which has both bound and continuum eigenstates, subjected to a harmonically oscillating potential. They reveal a very rich structure for the time evolution of even very simple quantum system. In particular the transition from a bound state to the continuum is seen to be much more complex than the simple exponential decay obtained from conventional perturbation theory via Fermi's golden rule [2].

To obtain exact results we need to consider simplified model systems. In particular we cannot treat (for the present time) realistic description of the interaction between radiation and matter, such as the dipole approximation. We note however, that a comparison of our earlier results on an even simpler version of this model with experiments on the strong field ionization of Rydberg atoms [8] showed some surprising similarity between the two. We interpret this as an indication of a certain universality in the Schrödinger evolution of a system with bound and continuum spectrum subjected to time dependent external forces and that our model retains some of its behavior.

\section{The model}

The important idealizations in our model are: 1) Space is one-dimensional, 2) The "internal" potential creating the bound state is given by an attractive delta function at the origin, 3) The interaction with the external field has the form $\eta(t) U(x)$ where $\eta$ is periodic in time with a rectangular envelope, and 4) $U(x)$ is given by one or more delta functions at different locations on the $x$-axis. The first two assumptions are quite common for modeling short range binding potentials $[9,10]$ and should not affect greatly the basic physics of the ionization process. Assumption 3 means that we do not consider situations [11] where there is some "ramping" in turning the external field on and off. This should not be too serious when the pulse is of a long duration compared to the period of the field which is the case we are concerned with here. Assumption 4 on the other hand clearly makes the interaction in our model very different from the real interactions between radiation and matter: dissociation due to electromagnetic fields are described approximately by a dipole interaction of the form $U(x)=x$. Unfortunately we have not been able to obtain exact results for this case beyond those described in [11]. The only feature of the dipole interaction we are able to mimic is the spatial symmetry.

Using suitable units in which $\hbar=2 m=1$ ( $m$ is the particle mass) the time evolution of our system is given by the Schrödinger equation

$$
i \frac{\partial}{\partial t} \psi(x, t)=\left[-\frac{\partial^{2}}{\partial x^{2}}-2 \delta(x)+U(x) \eta(t)\right] \psi(x, t),
$$

where $\eta(t)=r \sin \omega t$ and the parameters $r, \omega$ represent the amplitude and frequency of the time dependent potential. The spatial structure of the external potential will be taken in two forms: $U_{1}(x)=2 \delta(x-a)$ and $U_{2}(x)=2[\delta(x+a)-\delta(x-a)] ; U_{2}$ has the symmetry of the dipole interaction. The factor 2 in front of the binding potential is chosen so that the unique bound state of the unperturbed system is $u_{b}(x)=e^{-|x|}$ with binding energy $E_{0}=\omega_{0}=1$. Eq.(1) is to be solved subject to the initial 
condition $\psi(x, 0)=u_{b}(x)$. We can readily extend our methods to more general sums of delta functions. Our main interest is in the survival probability of the bound state at time $t:|\theta(t)|^{2}=\left|\left\langle\psi(x, t) u_{b}(x)\right\rangle\right|^{2}$.

The case $U_{1}(x)$ with $a=0$, which corresponds the parametric perturbation of the binding potential, was treated in $[12,13]$. We showed there that $|\theta(t)|^{2}$ has both exponential and power law parts which are well separated only when the strength of perturbation $r$ is small. This was true for all $\omega$ away from resonances ( $\omega \not N^{-1}, N$ an integer), with $\omega<1$ corresponding to ionization via "multi-photon" processes. We also obtained there non-monotonic dependence of the escape rate on $r$ and $\omega$. Somewhat to our surprise we found qualitative (and even semi-quantitative) agreement between the predictions of this model about resonance behavior of the survival probability of localization and some experimental observations on the ionization of Rydberg atoms by strong microwave fields [8]. It was also proved in [13] that when $\eta(t)$ is a sum of a finite number of harmonics, $\left(\sum_{j=1}^{M} A_{j} e^{i j \omega t}+\right.$ complex conjugate $)$, then the survival probability $|\theta(t)|^{2} \rightarrow 0$ as $t \rightarrow \infty$ for any $M<\infty, A_{M} \neq 0$. There are however very special infinite sequences $A_{j}$, given explicitly in [13], for which we proved that the system never ionizes fully $|\theta(t)|^{2} \nrightarrow 0$.

Here we show that the situation is quite different and much richer when we consider $U_{1}(x)$ with $a \neq 0$ or $U_{2}(x)$. (In physical units the position of perturbation corresponds to $\hbar a / \sqrt{2 m E_{0}}$ ). In particular we prove that for $\eta(t)=r \sin \omega t$ there exist two-dimensional manifolds in the space of the three parameters $\omega, r, a$ on which $|\theta(t)|^{2} \nrightarrow \rightarrow 0$ as $t \rightarrow \infty$. (We shall take without loss of generality $\omega, r, a$ positive.) This means that while $|\theta(t)|^{2} \rightarrow 0$ for almost all parameter values of the forcing, "exceptional" cases can also be constructed quite readily. This does not occur for fixed $\omega$ and $a$ if $r$ is small enough and is thus outside conventional perturbation theory. We find in addition that when $\omega$ is very close to a resonance, $\omega \approx N^{-1}+$ dynamic Stark shift, then the decay may not have the exponential part predicted by the golden rule no matter how small $r$ is even when $|\theta(t)|^{2} \rightarrow 0$.

We can also consider the case when there is no binding potential at all, i.e. the term $-2 \delta(x)$ is absent in (1). In this case we have for $\eta(t)=0$ a free particle, which when initially localized in the vicinity of the origin will diffuse away: the probability of being in any fixed region decaying as $t^{-1}$. On the other hand the perturbations with special values of $a, \omega, r$ can make the particle stay localized for all time.

\section{Results for $U_{2}(x)$}

We give here an outline of the proof which follows along the lines presented in detail for the case $a=0$ in [13]. Expanding $\psi(x, t)$ in terms of the eigenfunctions of the unperturbed Hamiltonian $H_{0}=-\frac{d^{2}}{d x^{2}}-2 \delta(x)$, we write

$$
\psi(x, t)=\theta(t) e^{-|x|+i t}+\int_{-\infty}^{\infty} \Theta(k, t) u(k, x) e^{-i k^{2} t} d k
$$

where the initial conditions are $\theta(0)=1, \Theta(k, 0)=0$ and the explicit expression [12] for the continuum states are

$$
u(k, x)=\frac{1}{\sqrt{2 \pi}}\left(e^{i k x}-\frac{e^{i|k x|}}{i+i|k|}\right), \quad-\infty<k<\infty .
$$

Substituting (2) into (1) we obtain $\psi(x, t)$ in the form of a functional of $\psi(a, t)$ and $\psi(-a, t)$ while $\theta(t)$ can be written as

$$
\theta(t)=1+2 i e^{-a} \int_{0}^{t} \eta(s)\left[Y^{+}(s)-Y^{-}(s)\right] d s,
$$

where $Y^{ \pm}(t)=e^{-i t} \psi( \pm a, t)$. We now express $\Theta(k, t)$ in terms of $Y^{ \pm}(t)$ too, take $x= \pm a$ in (2) and obtain a coupled pair of integral equations

$$
Y^{ \pm}(t)=e^{-a}+\int_{0}^{t} \eta(s)\left[K^{ \pm}(t-s) Y^{+}(s)-K^{\mp}(t-s) Y^{-}(s)\right] d s .
$$

The Laplace transforms of the kernels $K^{ \pm}(t)$ are

$$
k^{-}(p)=\frac{e^{-2 a \sqrt{1-i p}}}{\sqrt{1-i p}-1}, k^{+}(p)=\frac{1+k^{-}(p)}{\sqrt{1-i p}},
$$

with the choice of branch $\sqrt{1-i p} \rightarrow 1$ when $p \rightarrow 0$. For $\eta(t)=r \sin \omega t$, letting $y^{ \pm}(p)$ be the Laplace transforms of $Y^{ \pm}(t)$ and setting $f(p)=y^{+}(p)-y^{-}(p)$, eq.(3) yields

$$
\theta(t)=\frac{r e^{-a}}{2 \pi i} \int_{C} \frac{e^{p t}}{p}[f(p-i \omega)-f(p+i \omega)] d p
$$

with integration along a contour $C$ which goes from $-i \infty$ to $i \infty$ in the right half plane avoiding $p=0$ by a small semi-circle in the left half plane.

Survival of bound state: The survival probability $|\theta(t)|^{2}$ is determined (see (5)) by the analytic structure of $y^{ \pm}(p)$. Setting $y_{n}^{ \pm}=y(p+i \omega n), k_{n}^{ \pm}=k^{ \pm}(p+i \omega n)$ eqs.(4) turn into the recurrence relations for the vectors $y_{n}=$ $\left\{y_{n}^{+}, y_{n}^{-}\right\}, n \in \mathbf{Z}$

$$
y_{n}=\frac{e^{-a}}{p+i \omega n}\left(\begin{array}{l}
1 \\
1
\end{array}\right)-\frac{i r}{2}\left(\begin{array}{ll}
k_{n}^{+} & -k_{n}^{-} \\
k_{n}^{-} & -k_{n}^{+}
\end{array}\right)\left(y_{n-1}-y_{n+1}\right),
$$

where $y_{n}, k_{n}^{ \pm}$may be viewed as functions of the parameter $p$ in the strip $\Im(p) \in[0, \omega)$. The poles of $y(p)$ are in the left half-plane at $\xi_{0}+i \omega n, \xi_{0} \leq 0$, and the branch points at $p=-i-i n \omega, n \in \mathbf{Z}$ (the latter ones are inherited from $\left.k_{n}^{ \pm}(p)\right)$. After making horizontal cuts at $p=x+i n \omega,-\infty<x=\Re p \leq 0$ we push the contour $C$ in (5) along the branch cuts into the left half plane. The poles then contribute a series of residues with the common exponential factor $e^{\xi_{0} t}$ while the integrals around the cuts generate a contribution in the form of a series in 
terms of $t^{-j-1 / 2}, j \geq 1$ (see [12]). The imaginary part of $\xi_{0}, \Im \xi_{0}$, is identified as the dynamical Stark shift $[2,7]$ of the resonance frequency and $\Gamma=-2 \Re \xi_{0}$ is the decay exponent in the initial stage of evolution when $r$ is small and the exponential and polynomial parts of $|\theta(t)|^{2}$ may be separated.

If $\Re \xi_{0}=0$, i.e. the poles lie on the imaginary axis, then $\theta(t) \nrightarrow \rightarrow 0$ as $t \rightarrow \infty$. This can happen when on the imaginary $p$ axis the homogeneous recurrence,

$$
z_{n}=-\frac{i r}{2}\left(\begin{array}{cc}
k_{n}^{+} & -k_{n}^{-} \\
k_{n}^{-} & -k_{n}^{+}
\end{array}\right)\left(z_{n-1}-z_{n+1}\right),
$$

associated with (6), has non-trivial solutions which decay sufficiently rapidly as $n \rightarrow \pm \infty$, i.e. $\sum\left|z_{n}\right|^{2}<\infty$. This is a manifestation of the Fredholm alternative [14]. We show now that unlike the case $a=0$ treated in $[12,13]$ such solutions though non-generic are possible for $U_{2}(x)$ and for $U_{1}(x)$ with $a \geq 1 / 2$.

Setting $p=i g$ (with a real $g$ ) we construct a particular solution of (7) for $\omega>1$. It is clear that if $z_{j}=z_{j+1}=0$ then all successive $z_{n}$ will be zero too until the matrix in (7) becomes degenerate. We set $z_{n}=0$ for all $n \leq 0$ and require the determinant $\left(k_{0}^{+}\right)^{2}-\left(k_{0}^{-}\right)^{2}$ to vanish, which allows $z_{1} \neq 0$, in particular $z_{1}^{+}=z_{1}^{-}$. This implies

$$
a \sqrt{-1-g_{0}}=\pi N, \quad N=1,2, \ldots,
$$

where the parameter $g_{0} \in(-\omega,-1)$ represents the "binding energy" of a localized state produced by the perturbation (Floquet state [6,7]). For $n \geq 1$ all $k_{n}^{ \pm}$are real positive and the matrices in (7) are non-degenerate. By inverting them it is easy to show that $z_{n}^{-}=(-1)^{n+1} z_{n}^{+}$ and obtain a scalar recurrence for $z_{n}^{+}, n \geq 0$. Using a new variable $\rho_{n}=i z_{n+1}^{+} / z_{n}^{+}$the recurrence (7) takes the form

$$
\rho_{n}=\frac{2}{r k_{n}}-\frac{1}{\rho_{n-1}}, \rho_{1}=\frac{2}{r k_{1}},
$$

where $n>1$ and $k_{n}=k_{n}^{+}+(-1)^{n+1} k_{n}^{-}$.

A careful analysis for the case $N=1$ in (8) shows that the decaying solutions of (9) can be constructed with a unique $r=r(a, \omega)$. Two inequalities,

$$
k_{1} k_{2} \geq 2 k_{3}\left(2 k_{3}-k_{2}\right), \quad k_{1} k_{2} \geq k_{3}\left(4 k_{3}-k_{2}\right),
$$

which are necessary and sufficient for the existence of solutions of (9), specify regions in the $a, \omega$, plane. Relations (10) can always be satisfied if we choose $g_{0}+\omega \ll 1$ which makes $k_{1}$ large. The second of them gives the upper bound on $\omega$ which becomes very strict, $0<\omega+g_{0} \ll 1$ when $-g_{0} \sim 1$, i.e. $a$ is large, see (8). The interval where the stabilizing $r$ is located can be specified too and we can prove that for an arbitrary frequency $\omega>1$ there is an interval of $a=\pi / \sqrt{-1-g_{0}}$ with $g_{0}$ in $(-\omega,-1)$ where, for a particular choice of amplitude $r=r(a, \omega)$ the system does not ionize completely. Instead $\theta(t) \rightarrow e^{i g_{0} t} F\left(\omega_{s} t\right)$, where $\omega_{s}$ is the stabilizing frequency of the perturbation and $F$ is a periodic function with period $2 \pi$.

\section{Results for $U_{1}(x)$}

This type of stabilization of the bound state takes place for the perturbation with the potential $U_{1}(x)$ too, but only when $a \geq 1 / 2$. In this case there will again be a 2 -d manifold in $a, r, \omega$ variables for which the bound state is stabilized and $\theta(t) \rightarrow e^{i g_{0} t} F\left(\omega_{s} t\right)$, a quasi periodic function of $t$. We also computed $\theta(t)$ numerically for this model by solving the integral equation for $Y(t)$ and the most representative curves are shown in Fig.1. The slowest decay on the time interval $0<t \leq 160 \pi / \omega_{0} \approx 500$ was obtained for $\omega=1.12$ which is close to the value of $\omega_{s} \approx 1.089 \ldots$, evaluated by constructing the decaying solution of (9), and pushing it to as large $n$ as we can within the accepted precision. $|\theta(t)|^{2}$ near the Stark shifted resonance, $\omega=1.2$, has no interval in which the decay is exponential in contrast with such a decay observed for $\omega=1.25$ and $\omega=0.8$.

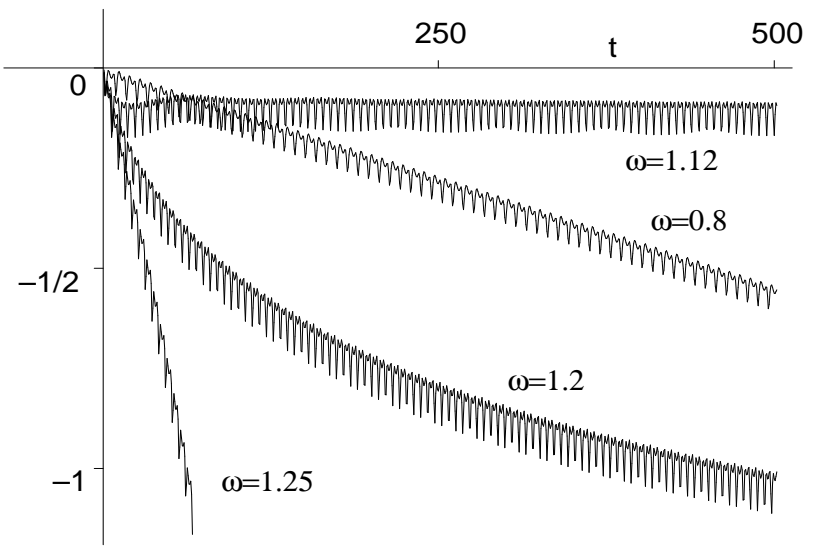

FIG. 1. Plot of $\log _{10}|\theta(t)|^{2}$ for $a \approx 0.59, r=1$.

The ripples on the curves in Fig. 1 have the frequency of the perturbation $\omega$ and the modulation is due to beats among $\omega, \omega_{0}$ and $g_{0}$.

A perhaps simplistic explanation of the stabilization is maybe to view it as some kind of bouncing of the trapped particle between the delta potential wells. Note that the eigenfrequency $g_{0}$ of the new bound state is a function (see (8)) of $a$ only, but the amplitude $r$ or the frequency $\omega$ must be fine tuned as functions of $g_{0}$ to prevent the leaking out of the particle wave function.

\section{Time decay of the bound state}

For the model with $U_{1}(x)$ the recurrence (6) has a scalar form and its solution can be written in terms of continued fractions which converge quite rapidly when $r$ is small. For $\omega>1$, neglecting terms of order of $r^{4}$ and higher, one may truncate the recurrence around each $n$ by taking $y_{n+m}=0$ if $|m| \geq 2$. The solution of the truncated system for $n=0$, which gives the main contribution to $\theta(t)$ has a pole at $\xi_{0}=O\left(r^{2}\right)$, that solves the equation

$$
1+\frac{r^{2}}{4} k_{0}^{+}\left(\xi_{0}\right)\left[k_{1}^{+}\left(\xi_{0}\right)+k_{-1}^{+}\left(\xi_{0}\right)\right]=O\left(r^{4}\right) .
$$

Using (11) the contour integration in (5), where $Y^{-} \equiv 0$, yields 
$\theta(t) \approx e^{\xi_{0}(\omega) t}+\frac{\omega e^{i(\omega-1-\Delta) t+i \pi / 4} \Re \xi_{0}(\omega)}{\sqrt{\pi}\left[\left(\omega^{2}-(1+\Delta)^{2}\right][(\omega-1-\Delta) t+1]^{3 / 2}\right.}$,

where $\Re \xi_{0}(\omega)=-r^{2} \lambda(\omega) \sqrt{\omega-1-\Delta}, \Im \xi_{0}(\omega)=\Delta=$ $r^{2} \sigma(\omega)$, and $\lambda(\omega), \sigma(\omega) \neq 0$ are of order $e^{-2 a}$ when $a$ is large. $\Delta$ represents the Stark shift $[6,7]$.

The survival probability $|\theta(t)|^{2}$ has initially an exponential regime where it decays as $e^{-\Gamma t}$, with $\Gamma=-2 \Re \xi_{0}$ proportional to $r^{2}$ in agreement with Fermi's golden rule. As $t$ increases an increasingly important role is played by transitions back to the bound state with probability proportional to the density near the origin as given by the second term of (12). As a result, for $t \gg \Gamma^{-1}$, the survival probability follows the power law decay $|\theta(t)|^{2} \sim t^{-3}$, (see also $[4,13,15,16])$. The mathematical origin of this power law is the square root branch point at the bottom of the continuous spectrum. Note that this is much faster than when an initially localized free particle is permitted to evolve. The probability of it remaining localized then decays as $t^{-1}$ [17].

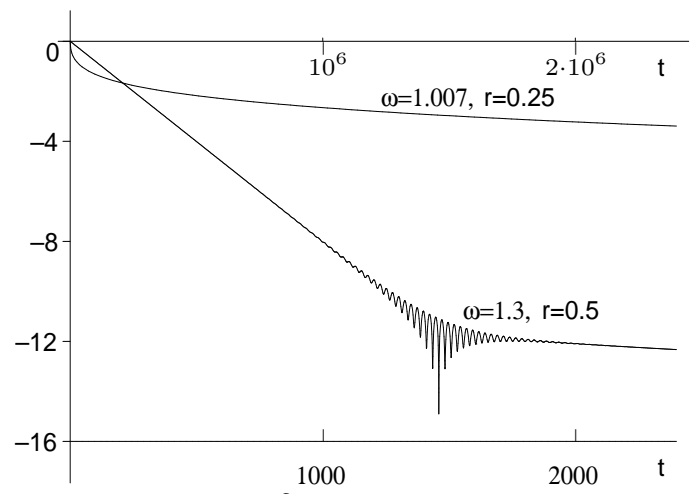

FIG. 2. $\log _{10}|\theta(t)|^{2}$ for two regimes of decay (plot of Eqs.(12),(14), note the different time scales and drastically different rates of decay). The upper curve for resonant $\omega$ does not follow Fermi's rule. The lower curve with the regular behavior (exponential with a $t^{-3}$ tail) is farther from resonance.

\section{Resonances}

Near the one photon resonance, $\omega \gtrsim 1$ (11) implies

$$
\xi_{0}(\omega)+r^{2} \lambda(1) \sqrt{\omega-1-\xi_{0}(\omega)}=i r^{2} \sigma(1)+O\left(r^{4}\right) .
$$

The solution of (13) gives $\Delta=O\left(r^{2}\right)$ and $\Gamma=o\left(r^{2}\right)$ whose dependence on $r$ is determined by the order in $r$ of $\omega-1$, but in the case when $\omega$ is very close to $1+$ $\Delta$ we cannot separate anymore the contributions in (3) because the poles of $y(p)$ are too close to the branch points. Integration in (5) yields

$$
\theta(t) \approx \frac{e^{i \epsilon t+i \pi / 4}}{\pi} \int_{-\infty}^{\infty} \frac{e^{-t r^{4} \lambda^{2}(1) x^{2} / 4} x^{2} d x}{\left[x^{2}-h(a)\right]^{2}+i x^{2}},
$$

where $h(a)=O(1)$ when $a$ is not large. For $r^{4} t \gg 1$ eq. (14) implies $|\theta(t)|^{2} \rightarrow t^{-3}$, but the initial exponential regime does not exist even for small $r$, see curves in Fig.2, which are constructed using (12)-(14). (Compare with $[4,15,16])$.

"Multiphoton" resonances. Let us compute the decay exponent when $\omega<1$ which corresponds to a "multiphoton ionization" $[6,8,12]$ for our simple model. To locate the singular point $p$ (which should be somewhere in the left half-plane very close to the imaginary axis) we require the homogeneous recurrence (a scalar analogue of (7)) to be solvable by a sequence $z_{n}$ such that $\left|z_{n}\right| \rightarrow 0$ when $|n| \rightarrow \infty$. Using (9) and the continued fractions we represent $\rho_{0}$ in terms of $\rho_{n}^{-1}$ for $n<0$ and $\rho_{n}$ when $n>0$. Both representations are rapidly convergent for $r \ll 1$ and the condition of their matching yields explicitly the solution for $\xi_{0}$ in the lowest order $\Delta=O\left(r^{2}\right)$ as before and $\Gamma=O\left(r^{2 N}\right)$. Zeros of $k_{-n}$ change the order of the decay exponent $\Gamma$ of the multiphoton ionization from $r^{2 N}$ to $r^{2 N+2}$ which drastically slows down the the ionization rate. The stabilization can also be expected for $\omega \approx 1 / 2,1 / 3, \ldots$ and therefore when $\omega<1 / 2$ we practically cannot see the decay if $t$ is not extremely large.

\section{Trapping of free particle}

Let us remove in (1) the binding potential and take the wave function

$$
\psi(x, 0)=\int_{-\infty}^{\infty} F(k) u(k, x) d k, \quad \int_{-\infty}^{\infty}|F(k)|^{2} d k=1,
$$

which describes a localized particle in the vicinity of the origin. Here $u(k, x)=(2 \pi)^{-1 / 2} e^{i k x}$ are the free eigenfunctions. For $\eta(t)=0$ the initial state evolves as

$$
\psi^{0}(x, t)=\int_{-\infty}^{\infty} F(k) u(k, x) e^{-i k^{2} t} d k \rightarrow t^{-1 / 2},
$$

when $t \rightarrow \infty$. Using in (1) the expansion in terms of $u(k, x)$ and Laplace transform $(\psi(x, t) \rightarrow \tilde{\psi}(x, p))$ we obtain the infinite set of equations

$$
\begin{gathered}
\tilde{\psi}_{n}(x)=\tilde{\psi}_{n}^{0}(x)+ \\
\frac{r}{2}\left[k_{n}^{+}(x)\left(y_{n-1}^{+}-y_{n+1}^{+}\right)-k_{n}^{-}(x)\left(y_{n-1}^{-}-y_{n+1}^{-}\right)\right],
\end{gathered}
$$

where $F_{n}(x)$ means $F(x, p+i \omega n), y_{n}^{ \pm}=\tilde{\psi}( \pm a, p+\omega n)$, and

$$
k^{ \pm}(x, p)=i \frac{e^{i|x \mp a| \sqrt{i p}}}{\sqrt{i p}}, \sqrt{i p} \rightarrow i \text { as } p \rightarrow i .
$$

Setting $x=a$ and $x=-a$ in (16) we obtain a recurrence similar to $(6)$ with vectors $\left\{\psi_{n}^{0}(a), \psi_{n}^{0}(-a)\right\}$. If on the imaginary axis the homogeneous recurrence (7) has a properly decaying solution for some $a, \omega, r$ then all $y_{n}$ have poles at $p=i g(a)+i \omega n$ respectively and therefore their inverse Laplace transforms which represent $\psi( \pm a, t)$ do not vanish as $t \rightarrow \infty$. For a given $\omega$ the requirement that the determinant $\left(k_{0}^{+}\right)^{2}-\left(k_{0}^{+}\right)^{2}$ vanishes gives the parameter $g_{0} \in(-\omega, 0)$ as a function of $a: g_{0}=-(\pi N / a)^{2}$ 
$(N$ is an integer and clearly $a>\pi / \sqrt{\omega})$. Setting $z_{n}=0$ for all $n \leq 0$ and $z_{1}^{+}=z_{1}^{-}$, we can again invert the matrices in (7), obtain (9) and repeat the previous computation. The explicit form of the coefficients,

$$
k_{n}=\frac{1+(-1)^{n+1} e^{-2 a \sqrt{g_{0}+\omega n}}}{\sqrt{g_{0}+\omega n}},
$$

implies here too a possibility to find a decaying sequence $\rho_{n}$ and a rapidly decreasing set of $z_{n}^{ \pm} \sim r^{n} \omega^{-n / 2} / \sqrt{n !}$, as $n \rightarrow \infty$.

The poles of $y_{n}$ develop by (17) into poles of $\tilde{\psi}(x, p)$ at the same points $p=i g_{0}+i \omega n$. Therefore as $t \rightarrow \infty$ the wave function $\psi(x, t)$ will survive near the origin an can be represented as a series related to poles of (16) in Laplace space

$e^{i g_{0} t} \sum_{n=1}^{\infty}\left[e^{-|x-a| \sqrt{g_{0}+\omega n}} Q_{n}(\omega t)+e^{-|x+a| \sqrt{g_{0}+\omega n}} P_{n}(\omega t)\right]$,

where the coefficients $P_{n}, Q_{n}$ are periodic functions which decay rapidly as $n$ tends to $\infty$.

\section{Concluding remarks}

Our results show the richness of the structure exhibited by a simple toy model driven externally in the presence of a continuum. The survival probability can change greatly, including trapping in a localized state, as the parameters of the external forcing are varied. While not all the features of this simple model can be expected to be mirrored by real atoms driven by electromagnetic fields we believe that some features are rather universal. In particular the power law decay [16] and the Fermi golden rule violation at resonances are expected to occur quite generally. Even if the location of resonance is not on the real axis of the energy plane but has a small imaginary component and therefore the localized state is slowly decaying, the power law tail can compete with the exponent on the whole observable time interval.

\section{Acknoledgments}

We thank R. Barker, R. Schrader and A. Soffer for useful comments. Research supported by AFOSR Grant \# F49620-01-0154 and NSF Grants \# 0103807, 0100495.

[1] Atoms in Intense Laser Fields, Ed. M. Gavrila, Adv. Atom. Mol. Opt. Phys. Supplement 1, (Academic Press, San Diego 1992); Adv. Atom. Mol. Opt. Phys., Eds. B. Bederson and H. Walther, (Academic Press, San Diego 1995); Super-Intense Laser-Atom Physics, Eds. B. Piraux, A. L'Huillier, K. Rzażevsky, (Plenum Press, New York 1993).

[2] C.Cohen-Tannoudji, J.Dupont-Roc, and G.Grynberg, Atom-Photon Interactions (Wiley, New York, 1992).
[3] Multiphoton Ionization of Atoms, Eds. S. L. Chin and P. Lambropoulos, (Academic Press, Toronto, New York 1984);

[4] S.R.Wilkinson, C.F.Bharucha, M.C.Fischer, K.W.Madison, P.R.Morrow, O.Nlu, B.Sundaram, and M.G.Raizen, Nature 387, 575 (1997).

[5] R.M.Potvliege and R.Shakeshaft, Phys.Rev.A 40, 3061 (1989); S.Chelkowsky, A.D.Bandrauk, and P.B.Corkum, PRL 19, 2355 (1990).

[6] J.N.Bardsley and M.J.Comella, Phys.Rev.A 39, 2252 (1989); M.S.Pindzola and M.Dörr, Phys.Rev.A 43, 439 (1991).

[7] N.B.Delone and V.P.Krainov, Multiphoton Processes in Atoms, Springer-Verlag, Berlin-New York (1994); A.Maquet, Shih-I Chu and W.P.Reinhardt, Phys. Rev. A 27, 2946 (1983); W.R.Salzman, Phys.Rev.A 10, 461 (1974); L.Pan, K.T.Taylor, and C.W.Clark, Phys.Rev.A 43, 6272 (1991); G.Scharf, K.Sonnenmoser, and W.F.Wreszinski, Phys.Rev.A 44, 3250 (1991); A.Buchleitner, D.Delande, J.Zakrzewski, R.N.Mantegna, M.Arndt, and H.Walther, PRL 75, 3818 (1995).

[8] P.M.Koch, Acta Physica Polonica A 93 No. 1, 105 (1998); P.M.Koch and K.A.H.van Leeuwen, Phys.Reports 255, 289 (1995); T.J.Bensky, G.Haeffler, and R.R.Jones, PRL 79, 2018 (1997); F.Benvenuto, G.Casati, and D.L.Shepelyansky, Phys.Rev.A 45, R7670 (1992); F.Benvenuto, G.Casati, and D.L.Shepelyansky, Phys.Rev.A 47, R786 (1993).

[9] S.Geltman, J.Phys. B 10, 831 (1974); S.M.Susskind, S.C.Cowley, and E.J.Valeo, Phys.Rev. A 42, 3090 (1994); Yu.N.Demkov and V.N.Ostrovskii, Zero Range Potentials and Their Application in Atomic Physics (Plenum, 1988).

[10] S. Albeverio, F. Gesztesy, R. Hoegh-Krohn, and H. Holden, Solvable models in quantum mechanics, SpringerVerlag, New York-Berlin (1988).

[11] A.Fring, V.Kostrykin, and R.Schrader, J. Phys. B: At. Mol. Opt.Phys. 29, 5651 (1996); A.Fring, V.Kostrykin, and R.Schrader, J.Phys.A: Math.Gen. 30, 8559 (1997).

[12] O.Costin, J.L.Lebowitz, and A.Rokhlenko, J.Phys.A 33, $6311(2000)$.

[13] O.Costin, R. D. Costin, J.L.Lebowitz, and A.Rokhlenko, C.M.P. 221, 1 (2001).

[14] M.Reed and B.Simon, Methods of Modern Mathematical Physics (Academic Press, New York, 1972).

[15] G.Garcia-Calderon, J.L.Mateos, and M.Moshinsky, PRL 74, 337 (1995); A.Buchleitner, D.Delande and J.-C.Gay, J.Opt.Soc.B 12, 505 (1995). A.Soffer and M.I.Weinstein, Jour.Stat.Phys. 93, 359 (1998).

[16] A.Soffer and M.I.Weinstein, J.Stat.Phys. 93, 359 (1998).

[17] A.Rokhlenko and J.L.Lebowitz, J.Math.Phys. 41, 3511 (2000). 


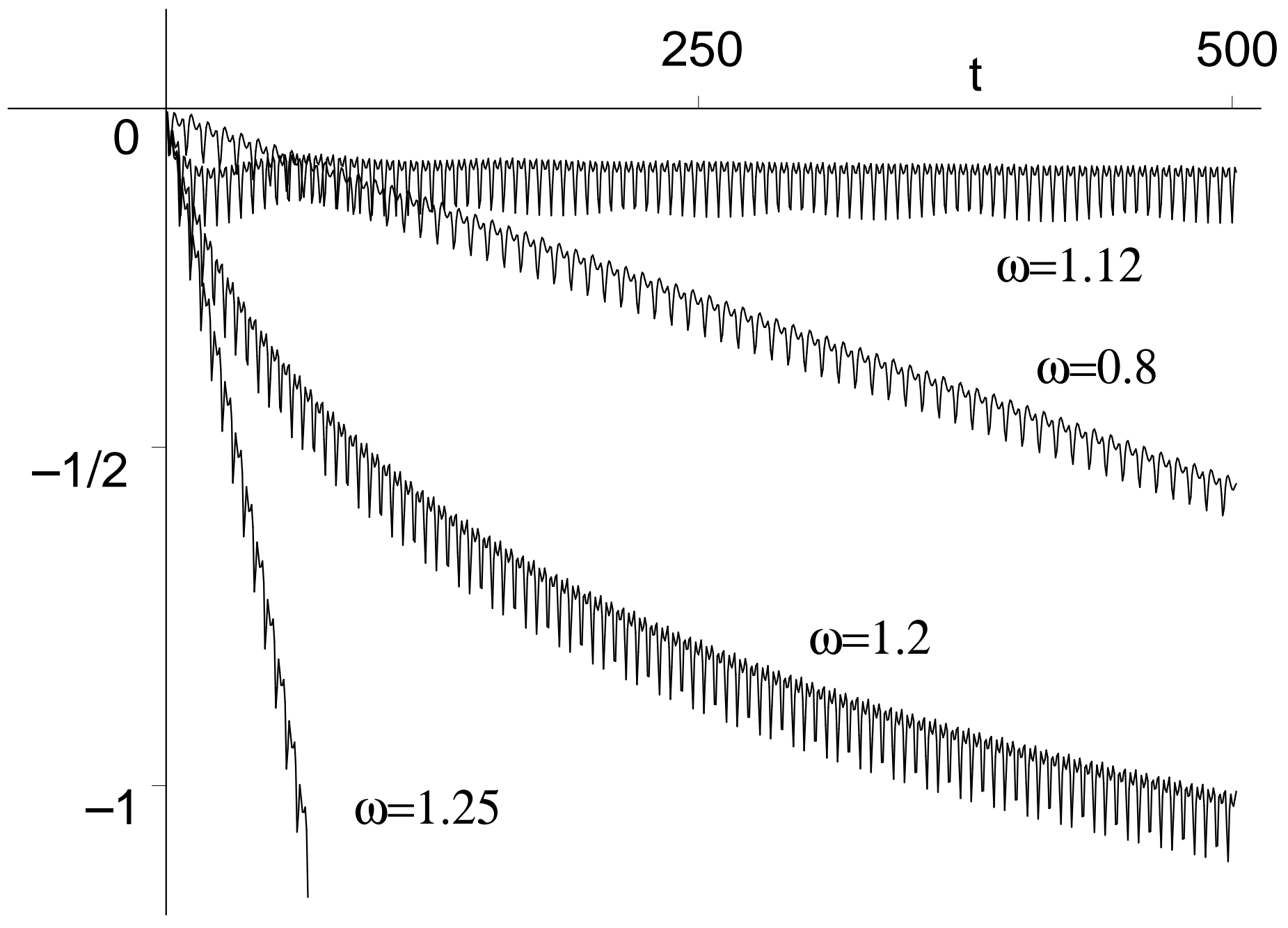




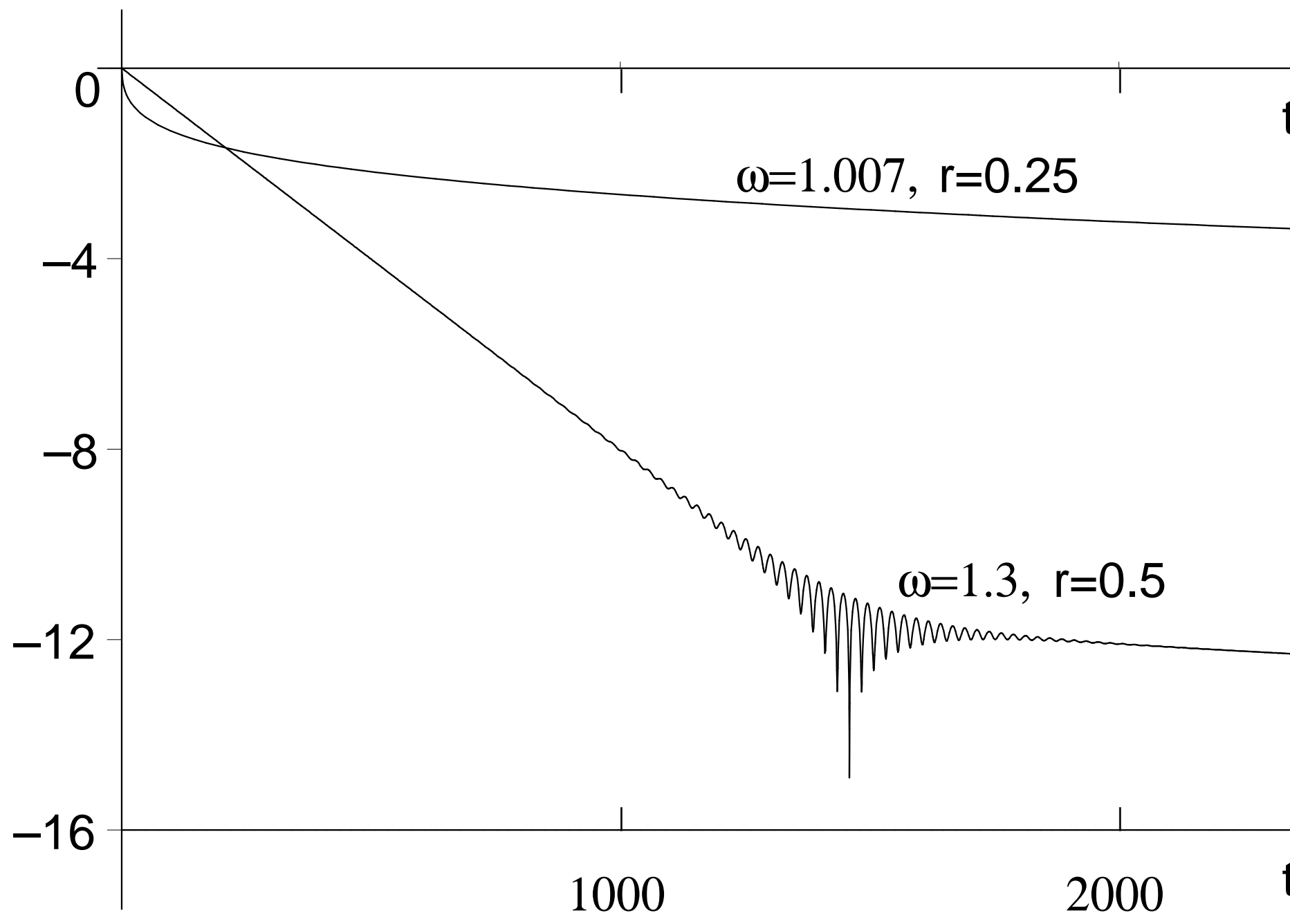

